\title{
THE CONDUCTION VELOCITY IN INTRACU- TANEOUS NERVE FIBRES OF THE FROG
}

\author{
YOSHIO TAKAGI* \\ Department of Physiology, Nagoya University School of Medicine
}

Studies on the afferent impulses and the sensory receptors in the skin have been made by many investigators $(1,2,3,4,5,6,9,14)$. Toad's skin afferent fibres were divided, according to the physiological property of the sensory unit, into five kinds, and the fibre size and the conduction rate of these five kinds of fibres were determined (13). The latencies for mechanical stimulation in the dorsal skin-nerve preparation of the frog (8) and for electrical pulse stimulation on the outside and inside of the calf skin-nerve preparation of the frog (7) were observed. The latencies thus measured include the true latency, or the time required for the initiation of nerve impulse, at the stimulated locus and its conduction time from sensory ending to recording electrode.

The conduction velocity of the intracutaneous part of cutaneous nerve fibres in the dorsal skin-nerve preparation of the frog was estimated by Kurashima (11), on the basis of the proportional relation of the latency to the travelling distance of the impulse in the skin. The experiments described in this paper are an extension of Kurashima's studies, worked by a similar method.

\section{METHODS}

The skin of the dorsum, abdomen and crus of the frog (Rana nigromaculata) was excised with its nerve and pinned out flat, the inside being placed upwards over a cork-plate. A nerve was placed on a pair of silver-silver chloride electrodes for recording afferent impulses. One electrode nearer to the skin was earthed and the other was led through the grid of a condenser coupled amplifier to a cathode-ray oscilloscope. The arrangement of the experimental apparatus is shown in fig. 1.

For mechanical stimulation was the mandril of an injection needle used, the tip of which was sharpened to a diameter of about $40 \mu$. The mandril was pushed down upon the skin by eight gram weight. The sweeping of the cathoderay was arranged to be synchronized with the stimulation. The artefact of the mechanical stimulation was intentionally marked in the cathode-ray oscillograph by means of connecting a condenser of $0.5 \mathrm{pF}$ between the mandril and the grid of the amplifier in order to indicate the moment that the mandril came in contact with the skin.

Received for publication April 2, 1958.

* 高木良雄 


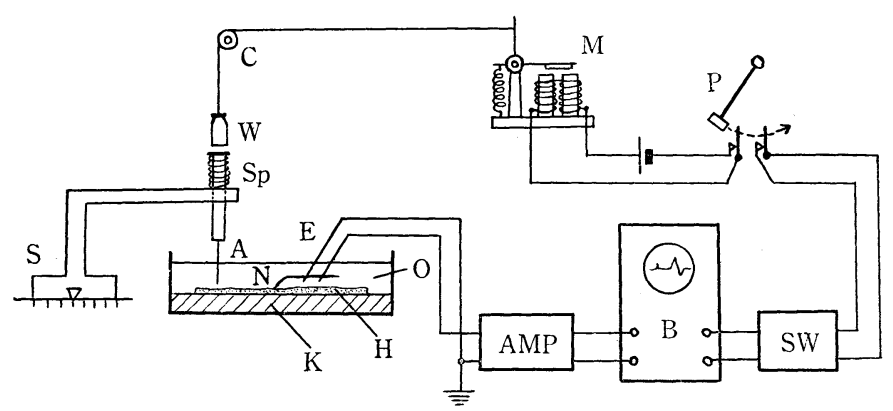

FIG. 1. The arrangement of the experimental apparatus. $A$ : mandril of an injection needle for mechanical stimulation, AMP : amplifier, $B$ : cathode-ray oscilloscope, $C$ : pulley, $E$ : recording electrodes, $H$ : skin, $K$ : cork-plate, $M$ : magnet, $N$ : cutaneous nerve, $O:$ oil, $P$ : pendulum for synchronizing magnet with sweeper, $S$ : scale, $S p$ : spring, $S W$ : sweeper, $W$ : weight.

The preparation including the recording electrodes was covered with oil in which the action potentials were recorded. The pathway of the cutaneous nerve fibres in the skin was, when necessary, observed by a binocular stereoscopic microscope at a magnification of $20 \times$ and photographed. The experiments presented were done at room temperature, which ranged from $24.0^{\circ}$ to $31.5^{\circ} \mathrm{C}$.

\section{RESULTS}

\section{1) Experiments in the dorsal skin-nerve preparations}

Spontaneous discharges were observed during about 120 minutes after setting the excised skin on the cork-plate. These discharges endured at times more than three hours. All of the present experiments were done after disappearance of the spontaneous discharges.

Two types of the afferent impulse volleys were found, the one was a rapidly adapting volley of large amplitude and the other a slowly adapting volley of smaller amplitude. The former were elicited mainly in the vicinity around the entering point of the given cutaneous nerve, while the latter at any place supplied by this nerve.

The duration of the latent period between the moment of stimulation and the onset of the first impulse volley was, as might be expected, minimum at the point close to the nerve entry and increased in general with the increase of the distance between the nerve entry and the stimulation spot. The latency measured here may include the following three time events: 1) the time required in the cutaneous receptor, that is a true latency, 2) the conduction time in the intracutaneous nerve fibre and 3) that in the extracutaneous nerve fibre. Of these three the first and the third may be rightly considered constant in the same preparation, thus the difference between any two latencies measured at a variety of distances may show the travelling time of the impulse volley in the skin. The latencies measured in one experiment, which are illustrated in fig. 2, were plotted against the distances between the nerve entry and the 

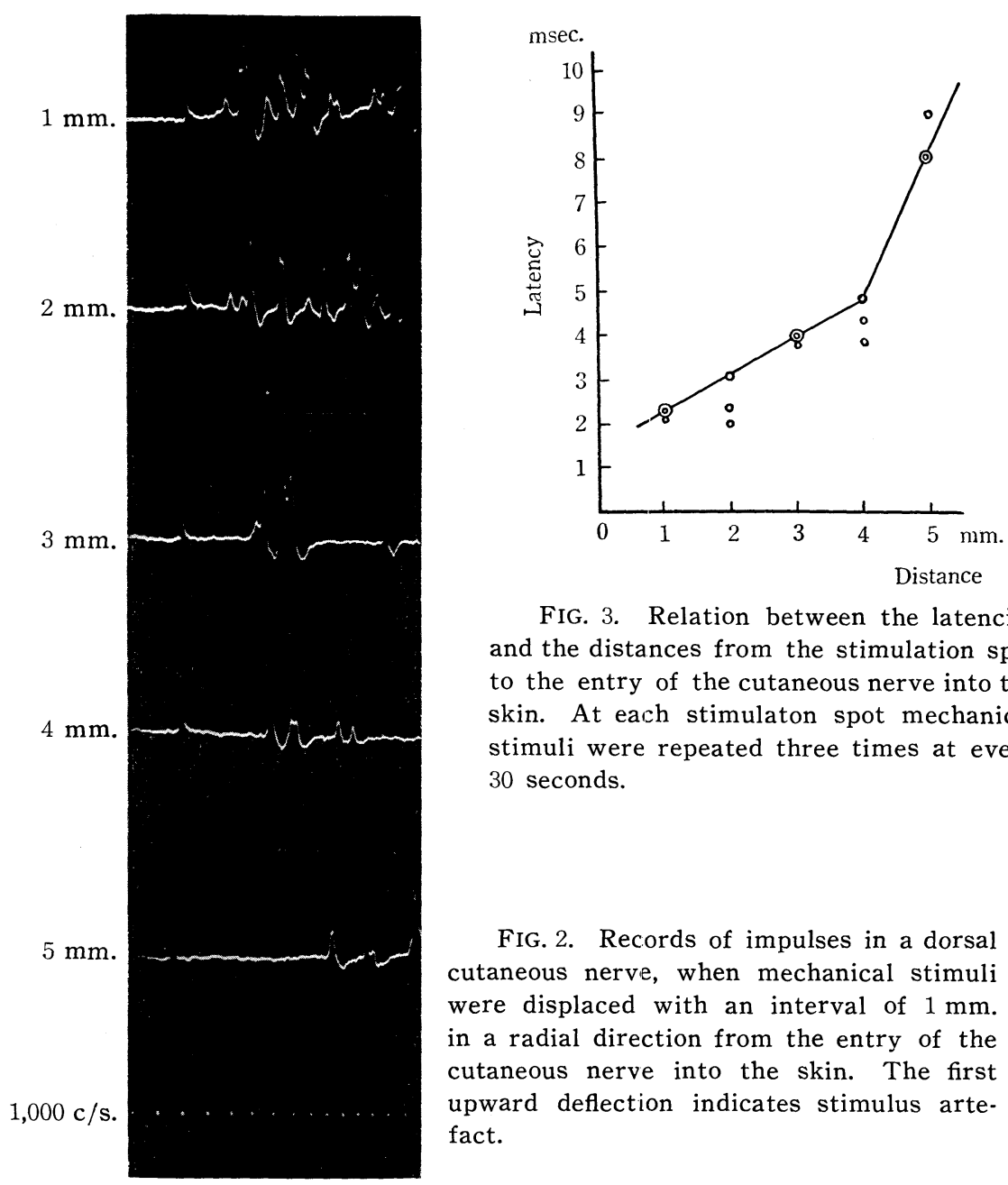

FIG. 3. Relation between the latencies and the distances from the stimulation spot to the entry of the cutaneous nerve into the skin. At each stimulaton spot mechanical stimuli were repeated three times at every 30 seconds.

FIG. 2. Records of impulses in a dorsal cutaneous nerve, when mechanical stimuli were displaced with an interval of $1 \mathrm{~mm}$. in a radial direction from the entry of the cutaneous nerve into the skin. The first upward deflection indicates stimulus artefact.

stimulation spot (fig. 3). The latency increased in avarage almost in a linear proportion to the distance. The magnitude of latency, however in some cases, fluctuated considerably in a wider range within the region of about $3 \mathrm{~mm}$. distant from the nerve entry, even when stimulated at the same point.

The conduction velocity in the intracutaneous nerve fibre calculated by the above procedure was $1.2 \mathrm{~m} / \mathrm{sec}$. in fig. 3 . The mean value in eight preparations presented was $1.47 \mathrm{~m} / \mathrm{sec}$. The latency increased abruptly at the point more than $4 \mathrm{~mm}$. distance, resulting in an abrupt decrease of the conduction velocity to $0.3 \mathrm{~m} / \mathrm{sec}$. in the case of fig. 3 . The abrupt decrease of conduction velocity was observed in half the experimental cases.

2) Experiments in the abdominal skin-nerve preparations

In the region innervated by ramus cutaneus abdominalis the latencies and 
therefrom the conduction velocities were measured, the stimulation point being displaced at an interval of $1 \mathrm{~mm}$. in medio-cranial, median and medio-caudal directions. In both medio-cranial and median directions the latencies increased in a rough proportion to the distances from the nerve entry, with the fluctuation ranging $1-3 \mathrm{msec}$. in the region less than $3 \mathrm{~mm}$., while the latencies in the region out of $3 \mathrm{~mm}$. were proved to be exactly proportional to the distances. The medio-caudal region, however, showed much larger fluctuation in latency, ranging from 0.8 to $3.5 \mathrm{msec}$. in the portion within the first $5 \mathrm{~mm}$. of its length and from 6 to $10 \mathrm{msec}$. in the portion beyond $5 \mathrm{~mm}$. and, therefore, no linear relationship between the latencies and the distances could be observed, so that the conduction velocity could not be estimated. The mean value of the conduction velocities of five cases, in which the proportional relation was proved, was $0.87 \mathrm{~m} / \mathrm{sec}$.

\section{3) Experiments in the crural skin-nerve preparations}

The crural skin was stimulated successively by moving the stimulating mandril with a micromanipulator, at each point which lined with a regular distance of $1 \mathrm{~mm}$., as shown with white dots in fig. 4 . Recording of action potentials was made from the ramus cutaneus cruris. The paths of the intracutaneous branches of this nerve could be traced macroscopically. One of these branches, which run relatively in a straight line from its entry, was chosen for the experiment, the other branches being excluded by incision at the points of their branching, as shown with two cuts at the right side in fig. 4. The latencies were brief along this remained branch and increased in general ac-

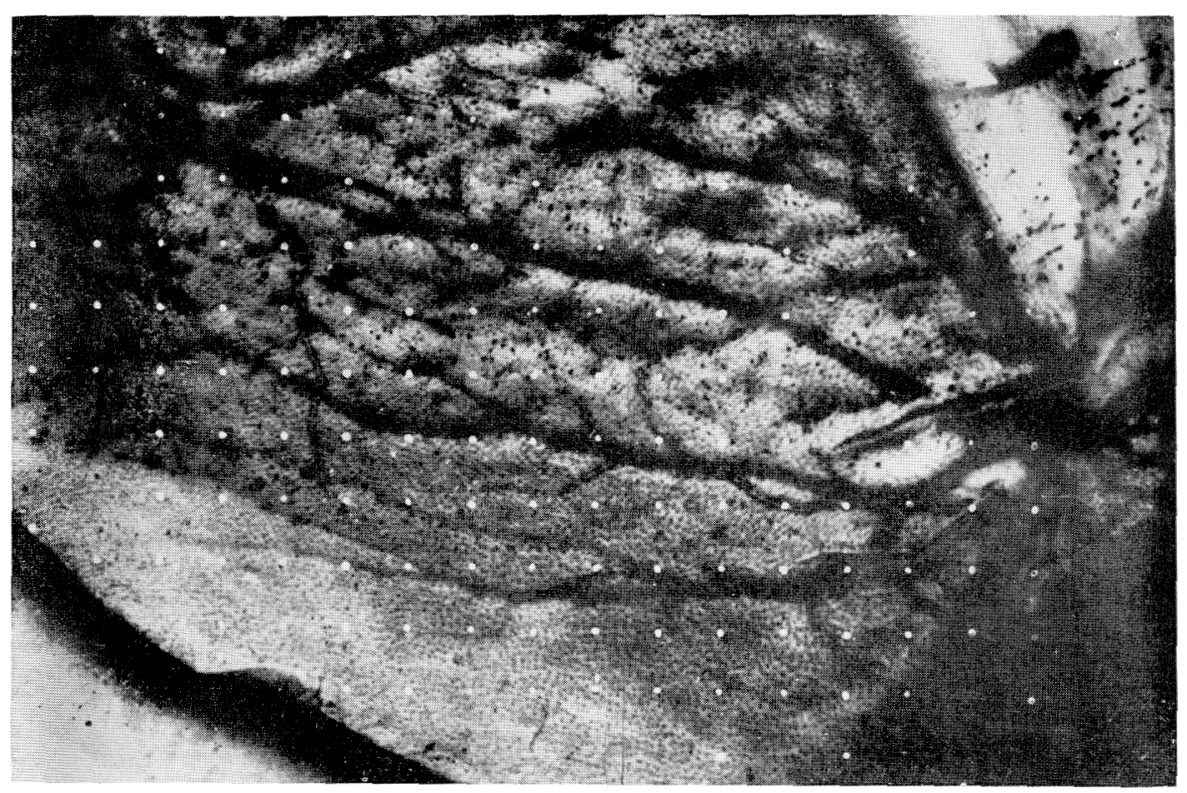

FIG. 4. Showing the stimulation spots, indicated at white dots, forming the checkers of 1 sq. mm., on the inner surface of the crural skin of the frog. 
cording as the stimulation spots were removed far from this branch, as illustrated in fig. 5, in which the spots of the same latency, e.g. the iso-latency points, were connected with a contour-line. Its distribution was in accord with the ramification of the intracutaneous branches. The intervals between each successive line became more narrow, as the lines were more distant from the branch chosen for the experiment, showing the decreasing of the conduction velocity with increasing the distance from it. According to the data obtained in this experiment, the velocity of conduction $(\mathrm{m} / \mathrm{sec}$.) varied from 0.26 to 5.00 in the portion of fibre of $1-2 \mathrm{~mm}$. in distance from the branching point, from 0.30 to 3.00 in that of $2-3 \mathrm{~mm}$., from 0.14 to 3.00 in that of $3-4 \mathrm{~mm}$. and from 0.22 to 1.20 in that of $4-5 \mathrm{~mm}$., resulting in the mean values of $1.32,0.74,0.59$ and 0.53 respectively. One determination made in the portion of 5-6 mm. showed a value of $0.36 \mathrm{~m} / \mathrm{sec}$. The conduction velocity along the branch was almost uniformly $10 \mathrm{~m} / \mathrm{sec}$. These results indicate that the conduction velocity in the intracutaneous nerve fibres is not uniform but decreases progressively towards the periphery of their distribution. It was furthermore noticed in this figure that there were several humps on the contour-lines which indicated the relative rapidness of conduction and were it was observed under a microscope that the second or the third order branches flowed in.

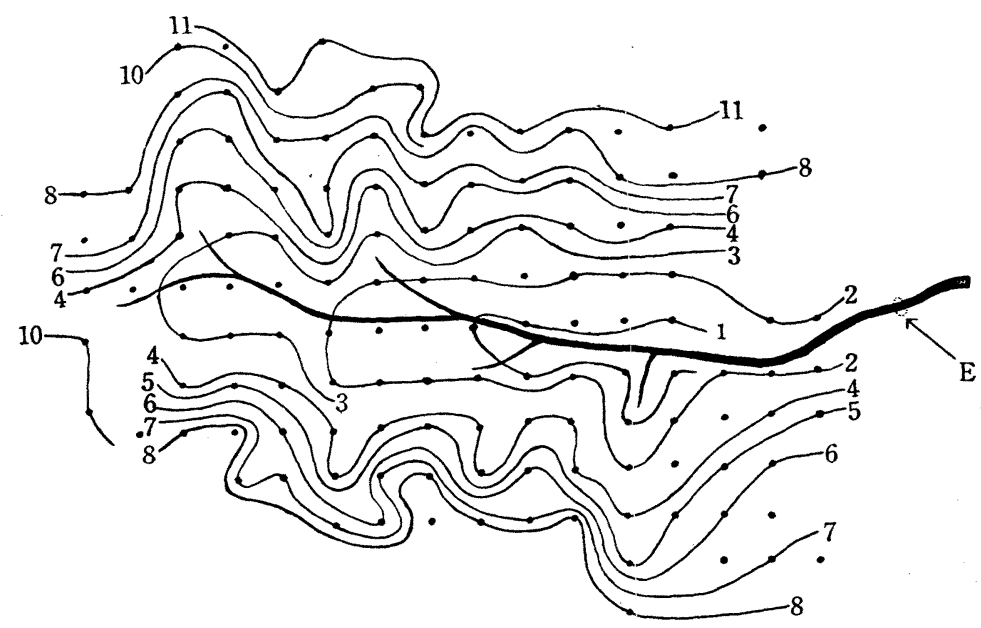

FIG. 5. Contour-lines of iso-latency, resulting from connecting the dots of the same latency with a line. The numbers on each line indicate the latency in millisecond. $E$ : the entry of the cutaneous nerve into the skin.

\section{DISCUSSION}

The difference in the effect of stimulation between the inside and the outside surface of the skin could not be found under this experimental condition that the excised skin-nerve preparation was placed on a cork-plate and pinned out flat without stretching intensely. This has also been demonstrated by Kura- 
shima (11). This experimental condition was taken because the fall in the threshold to mechanical stimulation with increase of stretching was greater for the outside than the inside, when the excised skin was sustained by clamping its peripheral ends between the two rings like an embroidery hoop, and because Loewenstein (12) reported that the stretching elicited the excitation of mechanoreceptors in the frog skin.

A receptive field for mechanical stimulation, according to Adrian et al. (4), was of exceedingly variable size ranging from 4 to 100 sq. $\mathrm{mm}$. in the dorsal skin of the frog. It, according to Kurashima (11), was within the extent of $6 \mathrm{~mm}$. from the nerve entry. In the experiments presented it was within 6-7 $\mathrm{mm}$. from the nerve entry in the dorsal skin of the frog and in the crural skin it was within about $7 \mathrm{~mm}$. from the intracutaneous branch which was traced macroscopically.

It is not certain whether the points of the skin stimulated in this experiments are innervated by a single nerve fibre, or not. Even if the innervation is single, impulses arising from the terminal of a fibre may be transmitted to its stem nerve branch entering the skin not always straightly, but may take a circuitous route as the presence of such circuitous courses of fibres has often been verified microscopically. The uncertainty of the proportional relationship between the latencies and the distances which was found in the portion of fibre within about $3 \mathrm{~mm}$. from the nerve entry may be probably due to such differences in the course of transmission. Microscopic observations revealed that, in the dorsal skin, almost all the cutaneous nerve fibres ran straightly in the radial direction from their stem branch to the ending in the skin, while, in the abdominal skin, they bended almost perpendicularly at a distance of about 5 $\mathrm{mm}$. from the entry of their stem branch. These findings are consistent with the fact that the proportional relationship between the latencies and the distances could be proved in the dorsal skin, but not always in the abdominal skin.

The velocity of conduction of nerve fibres in the dorsal skin decreased abruptly in the periphery over $5 \mathrm{~mm}$. distance from the nerve entry, and in the crural skin it decreased progressively with every millimeter from the intracutaneous branch chosen for the experiments. These results lead us to the conclusion that the conduction velocity is not uniform, but is smaller in the distal part than in the proximal part of the nerve fibres in the skin. Toad's skin afferent fibres were divided, by Maruhashi et al. (13), into five groups of varying sizes and it was demonstrated that the velocity of conduction decreases progressively as the diameter of fibre becomes smaller. The same conclusion was found by Hursh (10) and others. It may then be rightly presumed that conduction velocity is extremely slow in a ramified minute axon at the vicinity to a sensory terminal. Hitherto, receptor time, or true latency, was estimated on the basis of the assumption that the conduction velocity in the intracutaneous part is uniformly so fast as in the extracutaneous part of the cutaneous nerve fibre. And then the synaptic delay in the central nervous system has also been estimated under the premise of uniform conduction velocity along all nerve pathways. Now that the uneven velocity of conduction along a nerve 
fibre has been elucidated at least with skin nerves, this problem should be reconsidered more carefully.

\section{SUMMARY}

1. The intracutaneous afferent conduction for mechanical stimulation in skin-nerve preparations of the frog was studied, recording action potentials from cutaneous nerve fibres.

2. The latency from the beginning of stimulation to the onset of impulse volley increased in proportion to the distance between the entry of the cutaneous nerve into the skin and the stimulation spot in the dorsal and abdominal skin-nerve preparations.

3. The conduction velocity in the intracutaneous part of nerve fibre was estimated from the linear relation between the latency and the distance. The mean values of the conduction velocities in the dorsal and abdominal skin were 1.47 and $0.87 \mathrm{~m} / \mathrm{sec}$. respectively.

4. In the crural skin the conduction velocity was $10 \mathrm{~m} / \mathrm{sec}$. along the intracutaneous main branch of the cutaneous nerve, and decreased with increasing the distance from the stimulation spot to the intracutaneous branch concerned. The mean values of the conduction velocities at every millimeter distance from the intracutaneous branch were $1.32,0.74,0.59,0.53$ and $0.36 \mathrm{~m} / \mathrm{sec}$. respectively.

5. It may be concluded that the conduction velocity in the intracutaneous part of cutaneous nerve fibres is not uniform, but decreases progressively towards the periphery of its distribution.

The auther wishes to acknowledge the encouragement and guidance of Professor $\mathrm{K}$. Takagi during the course of this investigation.

The expenses of this work were in part defrayed by a grant from the Ministry of Education.

\section{REFERENCES}

1. AdRIAN, E. D. The Basis of Sensation. London: Christophers, 1928.

2. Adrian, E. D. Proc. Roy. Soc. B. 109 : 1, 1931.

3. Adrian, E. D. The Mechanism of Nervous Action. Philadelphia: University of Pennsylvania Press, 1932.

4. Adrian, E. D., Cattell, MCK. And Hoagland, H. J. Physiol. 72: 377, 1931.

5. Adrian, E. D. AND Zotterman, Y. J. Physiol. 61: 465, 1926.

6. CAtTell, MCK. AND HoAgland, H. J. Physiol. $72: 392,1931$.

7. Catton, W. T. J. Physiol. 136: 33 p, 1957.

8. Gray, J. A. B. And Malcolm, J. L. J. Physiol. 115: 1, 1951.

9. HogG, B. M. J. Physiol. 84: 250, 1935.

10. Hursh, J. B. Amer. J. Physiol. $127:$ 131, 1939.

11. Kurashima, S. J. Physiol. Soc. Jap. 18: 527, 1956.

12. Loewenstein, W. R. J. Physiol. 133: 588, 1956.

13. Maruhashi, J., Mizuguchi, K. And Tasaki, I. J. Physiol. $177:$ 129, 1952.

14. ZotTerman, Y. J. Physiol. $95: 1,1939$. 